\title{
Langmuir
}

pubs.acs.org/Langmuir

(C) 2009 American Chemical Society

\section{Construction of Metal-Ligand-Coordinated Multilayers and Their Selective Separation Behavior}

\author{
Guojun Zhang, ${ }^{*}{ }^{\dagger}$ Zhengang Ruan, ${ }^{\dagger}$ Shulan $\mathrm{Ji}^{,},{ }^{\dagger \dagger}$ and Zhongzhou Liu ${ }^{\star}$ \\ ${ }^{\dagger}$ Center for Membrane Technology, College of Environmental and Energy Engineering, Beijing University of \\ Technology, Beijing 100124, PR China and ${ }^{\star R e s e a r c h ~ C e n t e r ~ f o r ~ E c o-E n v i r o n m e n t a l ~ S c i e n c e s, ~ C h i n e s e ~}$ \\ Academy of Sciences, Beijing 100085, PR China
}

Received September 19, 2009. Revised Manuscript Received October 21, 2009

\begin{abstract}
In this article, a layer-by-layer ( $\mathrm{LbL}$ )-assembled coordination multilayer on planar and 3D substrates was explored by the alternate deposition of a transition-metal-containing polyelectrolyte and a ligand-containing polymer via the formation of complexes. The metal-ligand coordination between the building blocks of $\mathrm{Co}^{2+}$-exchanged poly(styrene sulfonate) (PSS) and poly(4-vinyl pyridine) (P4 VP) has been demonstrated using UV-vis, FTIR, and XPS. The film thickness, structure, and morphology as well as the wettability as a function of bilayer number have been systematically investigated by profilometry, SEM, AFM, and contact angle analyzers. For the purpose of separation applications, the metal-ligand-coordinated multilayer was assembled on both flat sheet and hollow fiber polymeric porous substrates using a dynamic pressure-driven LbL technique. It was demonstrated that the LbL-assembled PSS(Co $)_{1 / 2} / \mathrm{P} 4 \mathrm{VP}$ multilayer membrane had high dehydration performance with respect to different solvent-water mixtures; it also had aromatic compound permselectivity from aromatic-aliphatic hydrocarbons and water-softening capacity. Meanwhile, the successful assembly of multilayers on hollow fibers indicates that the dynamic pressure-driven LbL technique is a unique approach to the construction of multilayers on porous 3-D substrates. Therefore, the metal-ligand-coordinated self-assembly could emerge as a powerful technique for the preparation of a range of separation membranes in different types of modules.
\end{abstract}

\section{Introduction}

In recent years, there has been increasing interest in the construction of nanoscopically layered materials involving the layer-by-layer ( $\mathrm{LbL}$ ) sequential assembly of polymers on solid substrates. A variety of functional materials have been utilized in the LbL construction to make these structures suitable for prospective applications such as microcapsules, ${ }^{1,2}$ macroscopic sacs, ${ }^{3}$ sensitive solar cell films, ${ }^{4-6}$ biosensors, ${ }^{7-10}$ nanotube and nanocomposite materials, ${ }^{11-15}$ superhydrophobic and

*Corresponding authors. Fax: 86-10-67392393. E-mail: zhanggj@bjut. edu.cn (G.Z.), jshl@bjut.edu.cn (S.J.).

(1) Sagis, L. M. C.; Ruiter, R.; Miranda, F. J. R.; Ruiter, J.; Schroën, K.; Aelst,

A. C.; Kieft, H.; Boom, R.; Linden, E. Langmuir 2008, 24, 1608.

(2) Feng, Z.; Wang, Z.; Gao, C.; Shen, J. Adv. Mater. 2007, 19, 3687.

(3) Capito, R. M.; Azevedo, H. S.; Velichko, Y. S.; Mata, A.; Stupp, S. I. Science 2008, 319, 1812

(4) Zukalová, M.; Zukal, A.; Kavan, L.; Nazeeruddin, M. K.; Liska, P.; Grätzel, M. Nano Lett. 2005, 5, 1789.

(5) Luther, J. M.; Law, M.; Beard, M. C.; Song, Q.; Reese, M. O.; Ellingson,

R. J.; Nozik, A. J. Nano Lett. 2008, 8, 3488.

(6) He, J. A.; Mosurkal, R.; Samuelson, L. A.; Li, L.; Kumar, J. Langmuir 2003, $19,2169$.

(7) Shen, L.; Hu, N. Biomacromolecules 2005, 6, 1475.

(8) Camacho, C.; Matías, J. C.; Cao, R.; Matos, M.; Chico, B.; Hernández, J.; Longo, M. A.; Sanromán, M. A.; Villalonga, R. Langmuir 2008, 24, 7654.

(9) Gu, B. X.; Xu, C. X.; Zhu, G. P.; Liu, S. Q.; Chen, L. Y.; Wang, M. L.; Zhu,

J. J. J. Phys. Chem. B 2009, 113, 6553

(10) Lu, L. P.; Wang, S. Q.; Lin, X. Q. Anal. Chim. Acta 2004, 519, 161.

(11) Ai, S.; Lu, G.; He, Q.; Li, J. J. Am. Chem. Soc. 2003, 125, 11140.

(12) Shen, J.; Hu, Y.; Qin, C.; Ye, M. Langmuir 2008, 24, 3993.

(13) Xiong, H.; Chen, M.; Zhou, Z.; Zhang, X.; Shen, J. Adv. Mater. 1998, 10, 529 .

(14) Wang, C.; Wang, E.; Lan, Y.; Li, Q.; Mao, B.; Tian, C. Thin Solid Films 2008, 516, 6058 .

(15) Ramos, A. P.; Doro, F. G.; Tfouni, E.; Gonçalves, R. R.; Zaniquelli, M. E. D. Thin Solid Films 2008, 516, 3256.

(16) Ji, J.; Fu, J.; Shen, J. Adv. Mater. 2006, 18, 1441.

(17) Liao, K. S.; Wan, A.; Batteas, J. D.; Bergbreiter, D. E. Langmuir 2008, 24, 4245 . superhydrophilic surfaces, ${ }^{16,17}$ fuel cells, ${ }^{18,19}$ and separation membranes. ${ }^{20-27}$ In particular, LbL assembly has been widely recognized as an effective way to prepare permselective composite membranes, which are especially useful in the pervaporation separation of a wide variety of liquid mixtures such as azeotropes, close boiling-point isomers, and heat-sensitive mixtures, ${ }^{20-26}$ and they are of use in ion separation, ${ }^{27,28}$ water softening and desalination, ${ }^{29,30}$ and the size-selective separation of molecules (molecular sieving). ${ }^{31,32}$ A vast majority of LbL-assembled membranes have been fabricated on solid planar, ${ }^{20-22}$ tubular, ${ }^{23}$ or hollow fiber substrates. ${ }^{24-26}$ Recently, a pilot-scale hollow fiber pervaporation module with a length of $1 \mathrm{~m}$ was successfully produced in our laboratory. It suggested that the LbL-assembled membranes have great potential for industrial applications. ${ }^{26}$ However, in the blooming research area of LbL-assembled separation membranes, the driving force for the assembly applied so far is mostly the electrostatic force of aqueous-based oppositely charged polymers. In this case, those nonaqueous and

(18) Deligöz, H.; Yílmaztürk, S.; Karaca, T.; Özdemir, H.; Koç, S. N.; Össüzömer, F.; Durmus, A.; Gürkaynak, M. A. J. Membr. Sci. 2009, 326, 643.

(19) Zhao, C.; Lin, H.; Cui, Z.; Li, X.; Na, H.; Xing, W. J. Power Sources 2009, 194,168 .

(20) Zhu, Z.; Feng, X.; Penlidis, A. Mater. Sci. Eng., C 2006, 26, 1.

(21) Zhang, G.; Gu, W.; Ji, S.; Liu, Z.; Peng, Y.; Wang, Z. J. Membr. Sci. 2006, $280,727$.

(22) Zhang, G.; Yan, H.; Ji, S.; Liu, Z. J. Membr. Sci. 2007, 292, 1.

(23) Chen, Y.; Xiangli, F.; Jin, W.; Xu, N. J. Membr. Sci. 2007, 302, 78

(24) Zhang, G.; Gao, X.; Ji, S.; Liu, Z. Mater. Sci. Eng., C 2009, 29, 1877.

(25) Zhang, G.; Song, X.; Ji, S.; Wang, N.; Liu, Z. J. Membr. Sci. 2008, 325, 109.

(26) Zhang, G.; Wang, N.; Song, X.; Ji, S.; Liu, Z. J. Membr. Sci. 2009, 338, 43.

(27) Krasemann, L.; Tieke, B. Langmuir 2000, 16, 287.

(28) Hong, S. U.; Malaisamy, R.; Bruening, M. L. J. Membr. Sci. 2006, 283, 366.

(29) Hoffmann, K.; Tieke, B. J. Membr. Sci. 2009, 341, 261.

(30) Hashani, A. E.; Toutianoush, A.; Tieke, B. J. Membr. Sci. 2008, 318, 65.

(31) Jin, W.; Toutianoush, A.; Tieke, B. Appl. Surf. Sci. 2005, 246, 444.

(32) Liu, X.; Bruening, M. L. Chem. Mater. 2004, 16, 351. 
noncharged polymers cannot be used as the building blocks. Moreover, the driving force of electrostatic interaction is relatively weak. The stabilities of the resulting membranes are usually strongly dependent on the composition of the feed solution. For example, membranes with high water permselectivities such as water pervaporation, nanofiltration, and reverse osmosis generally contain hydrophilic materials that preferentially absorb water. Therefore, the building blocks of LbL-assembled membranes mostly originate from aqueous hydrophilic polymers. However, many hydrophilic polymers swell dramatically and are even destroyed in aqueous solutions to give large fluxes but little or no transport selectivity. ${ }^{33-35}$ Therefore, it is assumed that water swelling might be reduced if both aqueous and nonaqueous polymers are assembled on a substrate when membrane materials are selected. Generally, there is a compromise between hydrophilic and hydrophobic polymers. If coordination interaction between the transition metal and polymer is adopted as the driving force instead of electrostatic interaction, then the same stepwise assembly will yield a metal-ligand-coordinated multilayer, which will lead to a new class of membrane materials for use in separation. The advantage of these multilayers used for water pervaporation is that the water tolerance can be improved by coordinating a nonaqueous polymer. In fact, metal-ligand coordination has previously been applied to the assembly of other functional multilayers. For example, Zhang and co-workers reported that a PSS- $\mathrm{Cu}_{2} \mathrm{~S}$ nanoparticle/PVP hybrid film was successfully prepared by diffusing $\mathrm{H}_{2} \mathrm{~S}$ gas into a self-organizing multilayer of $\operatorname{PSS}(\mathrm{Cu})_{1 / 2} / \mathrm{PVP}$, which was constructed by taking advantage of the coordination between $\mathrm{Cu}^{2+}$ and pyridine. ${ }^{13}$ South et al. developed coordination multilayers consisting of $\mathrm{Pd}$ (II) pincer-type complexes and poly(vinyl pyridine) on quartz substrates. ${ }^{36}$ Rubenstein's group constructed gold nanoparticle mono- and multilayers on gold surfaces using $\mathrm{Zr}^{4+}$ coordination self-assembly. ${ }^{37}$ They further developed LbL branched multilayers on $\mathrm{Au}$ surfaces via $\mathrm{Zr}^{4+}$ metal-organic coordination. ${ }^{38}$ Recently, the divergent growth of surface-initiated dendritic nanostructures on gold surfaces in a very controlled, stepwise manner using metal-organic coordination as the binding motif was reported..$^{39}$ Abe et al. investigated the coordination molecular multilayers with high redox activity on an electrode surface. ${ }^{40}$ More recently, Northrop et al. reviewed state-of-the-art research with regard to the coordination-driven self-assembly of functionalized supramolecular metallacycles. ${ }^{41}$ Although there has been an accelerated development of LbL-coordination assembly, the selective separation properties of metal-ligand-coordinated multilayers remain largely unexplored. Meanwhile, a review of the literature reveals that most of the previous studies focus on the coordination self-assembled multilayer on rigid substrates such as quartz slides, silicon wafers, gold surfaces, and electrodes. ${ }^{13,36-41}$ However, for a permselective dense membrane, it is necessary to construct a permselective layer on a porous substrate. To obtain a defect-free dense membrane, traditional static LbL assembly is a

(33) Karakane, H.; Tsuyumoto, M.; Maeda, Y.; Honda, Z. J. Appl. Polym. Sci. $1991,42,3229$

(34) Xu, J. B.; Bartley, J. P.; Johnson, R. A. J. Appl. Polym. Sci. 2003, 90, 747.

(35) Kurkuri, M. D.; Aminabhavi, T. M. J. Appl. Polym. Sci. 2003, 89, 300.

(36) South, C. R.; Weck, M. Langmuir 2008, 24, 7506.

(37) Wanunu, M.; Popovitz-Biro, R.; Cohen, H.; Vaskevich, A.; Rubinstein, I. J. Am. Chem. Soc. 2005, 127, 9207.

(38) Wanunu, M.; Vaskevich, A.; Cohen, S. R.; Cohen, H.; Arad-Yellin, R.; Shanzer, A.; Rubinstein, I. J. Am. Chem. Soc. 2005, 127, 17877.

(39) Wanunu, M.; Vaskevich, A.; Shanzer, A.; Rubinstein, I. J. Am. Chem. Soc. 2006, 28,8341 .

(40) Abe, M.; Michi, T.; Sato, A.; Kondo, T.; Zhou, W.; Ye, S.; Uosaki, K.; Sasaki, Y. Angew. Chem., Int. Ed. 2003, 42, 2912.

(41) Northrop, B. H.; Yang, H.; Stang, P. J. Chem. Commun. 2008, 45, 5896.

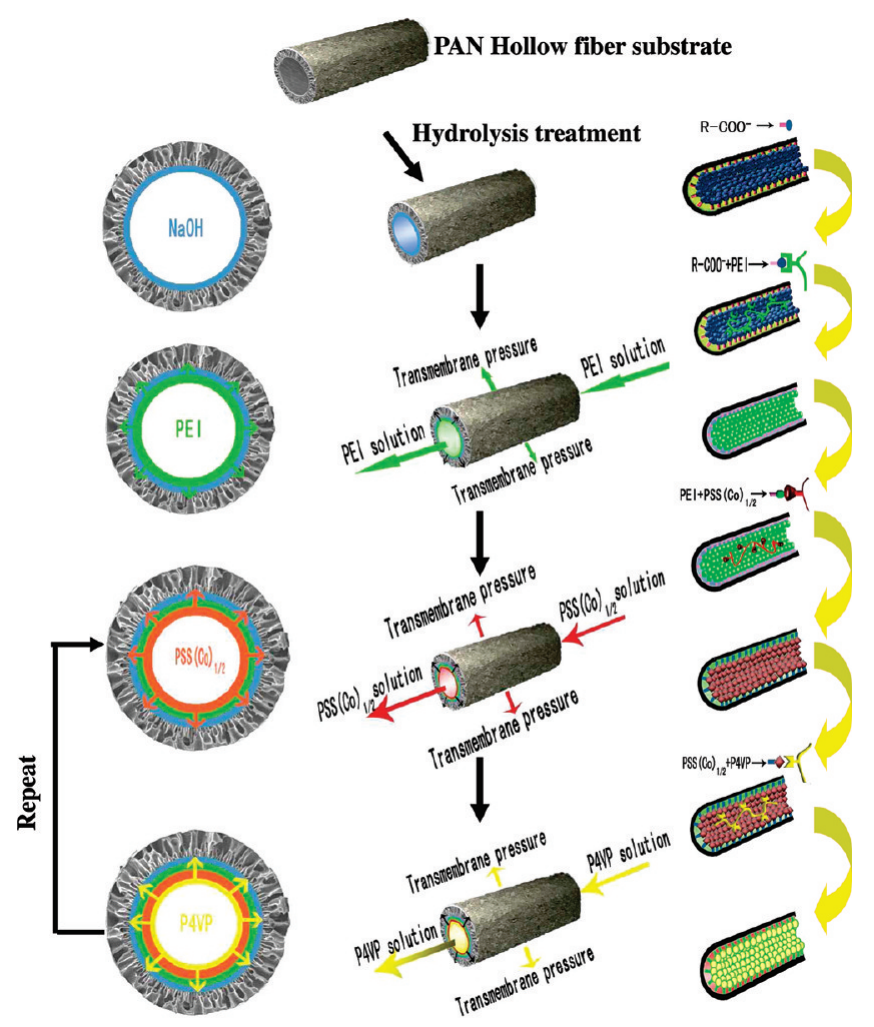

Figure 1. Schematic illustration of the preparation of a metalligand multilayer on a PAN hollow fiber substrate membrane by dynamic pressure-driven LbL assembly.

time-consuming process used to cover the defects of porous substrates. Moreover, how to fabricate the selective layer on a single side of the flat sheet and hollow fiber substrate membranes is important to improving selective transport for separation membranes and is also a difficult issue in the case of the traditional static LbL technique. In particular, it is difficult to construct multilayers on the inner surfaces of hollow fibers, which is a dominant and promising membrane module type in the area of separations.

Herein, we report on a metal-ligand-coordinated multilayer by alternatively depositing a transition-metal $\left(\mathrm{Co}^{2+}\right)$-containing polyelectrolyte and a ligand-(pyridine)-containing polymer via the formation of complexes. The PSS $(\mathrm{Co})_{1 / 2} / \mathrm{P} 4 \mathrm{VP}$ films were first prepared on quartz substrates to track the growth process via $\mathrm{UV}$-vis spectroscopy. The coordination role of cobalt ions in the assembly was confirmed using FTIR and XPS. In the second part, by using the porous characteristics of the ultrafiltration (UF) substrate, we used dynamic pressure-driven LbL to construct metal-ligand-coordinated selective multilayers on a single side of both flat sheet and hollow fiber polymeric substrates. It greatly reduced the number of deposition cycles compared with the number in the traditional static LbL process. The water-pervaporation behavior was subsequently examined with different organic solvent-water mixtures. The possible separation performance of an aromatic-aliphatic hydrocarbon and water desalination were also explored. In particular, a schematic representation of multilayer formation with $\mathrm{PSS}(\mathrm{Co})_{1 / 2}$ and $\mathrm{P} 4$ VP on a 3-D hollow fiber porous substrate is shown in Figure 1. As shown in Figure 1, the negative charge surface was first created by hydrolyzing PAN hollow fibers on the basis of the conversion of $-\mathrm{CN}$ groups on the PAN membrane surface into carboxyl groups under alkaline conditions. After precoating the poly(ethyleneimine) (PEI) layer, a $\mathrm{PSS}(\mathrm{Co})_{1 / 2} / \mathrm{P} 4 \mathrm{VP}$ multilayer could 
be successfully assembled onto the inner surface of hollow fibers under a certain transmembrane pressure. The creation of such films on polymeric porous substrates could provide a new approach to the formation of selective separation membranes in different module types, thus extending the usefulness of LbL assembly. Because our approach can be applied, in principle, to other multilayer assemblies, it will open significant possibilities for the rapid construction of new multilayers on various porous substrates, which will be of considerable use in many fields.

\section{Experimental Section}

Chemicals and Materials. Poly(sodium styrene sulfonate) (PSSNa, $\left.M_{\mathrm{w}} 70000\right)$ and poly(4-vinyl pyridine) (P4 VP, $M_{\mathrm{w}}$ $60000)$ were obtained from Aldrich. Branched poly(ethyleneimine) (PEI, $M_{\mathrm{w}} 60000$ ) was purchased from Acros. Sodium hydroxide, sodium chloride, magnesium sulfate, cobalt nitrate, benzene, cyclohexane, ethyl acetate, tert-butanol, isobutanol, butanol, 2-propanol, and ethanol were provided by the Beijing Chemical Factory. The chemicals were all used as received without further purification. Quartz and $\mathrm{CaF}_{2}$ substrates for UV-vis and FTIR measurements were purchased from Beijing Kinglass Quartz Co. Ltd. and Beijing East Chemical Industry Co., Ltd., respectively. Flat sheet PAN UF membranes with a nominal molecular weight cutoff of 30000-40000 were supplied by Sepro Membranes. Hollow fiber PAN substrate membranes were spun by a dry/wet-phase inversion method in our workshop. ${ }^{42}$ The inner diameter of each hollow fiber was $1.1 \mathrm{~mm}$.

Multilayer Buildup onto Quartz and $\mathbf{C a F}_{2}$ Slides. Quartz slides were treated with boiling piranha solution (30:70 v/v $\mathrm{H}_{2} \mathrm{O}_{2} /$ $\mathrm{H}_{2} \mathrm{SO}_{4}$ ) for $30 \mathrm{~min}$, followed by rinsing with copious amount of ultrapure water. The $\mathrm{CaF}_{2}$ slides were polished with talcum powder before being assembled. All substrates were first primed with a PEI layer by exposing them to a $0.25 \mathrm{wt} \%$ PEI aqueous solution for $30 \mathrm{~min}$, followed by extensive rinsing with water and drying with nitrogen. To prepare a metal $\left(\mathrm{Co}^{2+}\right)$-containing polyelectrolyte, $0.44 \mathrm{wt} \% \mathrm{Co}\left(\mathrm{NO}_{3}\right)_{2}$ was added to a $0.62 \mathrm{wt} \%$ PSSNa solution so that $\mathrm{Co}^{2+}$ could exchange with $\mathrm{Na}^{+}$of PSSNa in solution. P4 VP was dissolved in ethanol to a concentration of $0.23 \mathrm{wt} \%$. Because $30 \mathrm{~min}$ is a typical and commonly used deposition time in LbL self-assembled films, it was selected for subsequent experiments. The assembly of the PSS $(\mathrm{Co})_{1 / 2} / \mathrm{P} 4 \mathrm{VP}$ multilayer was conducted by consecutively immersing the PEIcoated substrates into the respective polymer solutions for $30 \mathrm{~min}$ and rinsing the adsorbed layers thoroughly with ultrapure water and ethanol. Prior to the next cycle, the films were dried with nitrogen. Alternating PSS $(\mathrm{Co})_{1 / 2} / \mathrm{P} 4 \mathrm{VP}$ multilayer films could be obtained by repeating these two steps in a cyclic fashion. Control experiments were conducted to ensure that there was no substantial difference among the parallel samples.

Multilayer Buildup onto Flat Sheet Hydrolyzed PAN Porous Substrates. To obtain a dense membrane for pervaporation separation and water desalination, metal-ligand-coordinated multilayers were assembled on a flat sheet hydrolyzed PAN porous substrate using a dynamic LbL method. On the basis of our previous study, the PAN UF membrane was hydrolyzed for $30 \mathrm{~min}$ at $65^{\circ} \mathrm{C}$ by immersing the membranes into a $2 \mathrm{~N}$ $\mathrm{NaOH}$ aqueous solution. ${ }^{22}$ The hydrolyzed membranes were rinsed with ultrapure water and then loaded into a dead-end filtration cell for dynamic assembly. The oppositely charged PEI was filtered to form the first precoated layer on the top surface. The membrane was then taken out, briefly rinsed with ultrapure water, and dried in an oven at $45^{\circ} \mathrm{C}$ for about $2 \mathrm{~h}$. Then the dried membrane was reloaded into the filtration cell. The $\operatorname{PSS}(\mathrm{Co})_{1 / 2}$ solution was filtered for $30 \mathrm{~min}$. Following the rinsing and drying steps, P4 VP ethanol solution was also filtered for $30 \mathrm{~min}$. The repetition of filtration procedures of $\mathrm{PSS}(\mathrm{Co})_{1 / 2}$ and $\mathrm{P} 4 \mathrm{VP}$

(42) Liu, Z.; Zhang, G.; Peng, Y.; Ji, S. Desalination 2008, 233, 55. solutions led to multilayer formation on the flat sheet PAN substrates. In each filtration step, the pressure was controlled at $0.1 \mathrm{MPa}$. The assembly was carried out at room temperature.

Multilayer Buildup onto Hollow Fiber Hydrolyzed PAN Porous Substrates. As illustrated in Figure 1, construction of the metal-ligand-coordinated multilayer onto a 3-D hollow fiber substrate was also performed. Similar to the flat sheet PAN membrane, the PAN hollow fibers were first hydrolyzed to obtain a negatively charged surface. The hydrolyzed fibers were then put into a polymethylmethcrylate tube with a diameter of $1.5 \mathrm{~cm}$. Both ends of the hollow fiber module were sealed with epoxy resin. The assembly experiments were carried out using a laboratoryfabricated cross-flow negative-pressure filtration cell, which has been previously reported. ${ }^{25}$ PEI was precoated onto the inner surface by recycling PEI aqueous solution in the inner channels of hollow fibers. On the outer-surface side, a vacuum pump was used to form a negative pressure of $-0.09 \mathrm{MPa}$. Subsequently, the $\operatorname{PSS}(\mathrm{Co})_{1 / 2}$ aqueous and P4 VP ethanol solutions were alternatively provided to the lumen side of hollow fibers and then recycled by using two peristaltic pumps through separate tubing. Dynamic assembly was also conducted at a negative pressure of $-0.09 \mathrm{MPa}$. Similarly, after the assembly of each PSS $(\mathrm{Co})_{1 / 2}$ and P4 VP layer, the membrane was rinsed with substantial amounts of ultrapure water and ethanol, respectively. The inner surface of the hollow fiber was dried by recycling filtered nitrogen gas. After the assembly of 2.0 bilayers of PSS $(\mathrm{Co})_{1 / 2}$ and P4 VP (i.e., 4 layers), the hollow fibers were stored for pervaporation tests.

Pervaporation and Desalination Experiments. Pervaporation measurements of the assembled flat sheet and hollow fiber membranes were conducted using two laboratory-fabricated pervaporation systems, which have been described in our previous studies. ${ }^{21,22,24-26}$ It is notable that a specially designed cross-flow system was used to evaluate the hollow fiber multilayer membrane performance. $^{25}$ The feed solution was in contact with the inner surfaces of the hollow fiber modules at $60^{\circ} \mathrm{C}$. Three modules with same fabrication and modification conditions were examined for each pervaporation condition. The permeate vapor was trapped with liquid nitrogen. The downstream pressure was about $100 \mathrm{~Pa}$. Fluxes were determined by measuring the weight of liquid collected in the cold traps for a certain time under steady-state conditions. The compositions of feed solutions and permeates were determined using a gas chromatograph (GC-14C, Shimadzu). The separation factor $\alpha$ was calculated according to the quotient of the weight ratio of components $i$ and $j$ in the permeate, $Y_{i} / Y_{j}$, and in the feed, $X_{i} / X_{j}$.

$$
\alpha=\frac{Y_{i} X_{j}}{Y_{j} X_{i}}
$$

Desalination experiments were carried out using a crossflow membrane cell. The membranes with an effective area of $21.23 \mathrm{~cm}^{2}$ were loaded into the system for filtration. The feed solution was pressurized with a plunger pump. Both the retention and permeate streams were recycled to a feed tank. Filtration experiments with mixed electrolyte solutions containing $\mathrm{MgSO}_{4}$ and $\mathrm{NaCl}$ were implemented to determine the salt rejection. The filtration pressure was maintained at $0.6 \mathrm{MPa}$. The ion concentrations were measured using an ion chromatograph (ICS-1000, Dionex). The retention ratio was calculated on the basis of the data for the ion concentration in the feed and permeate solutions. The permeability $F$ was calculated by

$$
F=\frac{W}{A t \Delta P}
$$

where $W(\mathrm{~kg})$ is the total mass of permeate collected under transmembrane pressure $P(\mathrm{MPa})$ on a time scale $t(\mathrm{~h})$ and $A$ is the effective area of the membrane $\left(\mathrm{m}^{2}\right)$.

Characterization. The UV-vis absorption spectra of the multilayers on quartz slides were recorded using a spectrophotometer 


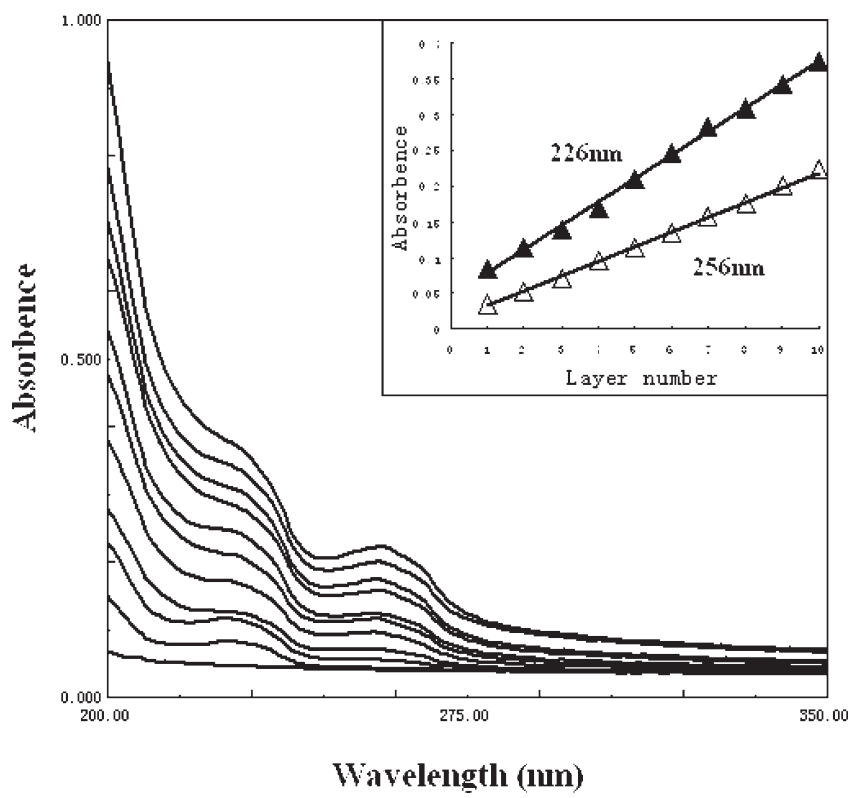

Figure 2. UV-vis spectra of $\left(\mathrm{PSS}(\mathrm{Co})_{1 / 2} / \mathrm{P} 4 \mathrm{VP}\right)_{n}$ films with $n=$ $1-10$ on quartz substrates (from bottom to top). The inset shows the plots of the absorbance values at 226 and $256 \mathrm{~nm}$ versus the number of bilayers.

(UV-2550, Shimadzu). Uncoated quartz slides were used as a reference spectrum. The film thickness was measured using a XP-1 profilometer (Ambios). A groove in the film was made using a razor blade, and the profilometer stylus measured the depth of the groove to give the film thickness. The thickness was measured three times at different locations, and the average was used as a data point. Fourier transform infrared (FTIR) spectra were obtained using a Vertex-70 spectrophotometer (Bruker, Germany). X-photoelectron spectroscopy (XPS) was used to confirm the composition of the multilayers. XPS measurements were carried out on a Thermo Fisher Scientific ESCALAB 250 spectrometer using a $\mathrm{Mg} \mathrm{K} \alpha \mathrm{X}$-ray source. A scanning electron microscope (SEM) (Hitachi-4700, Japan) was used to observe multilayer morphologies assembled on different substrates. For the multilayers on flat sheet and hollow fiber PAN substrates, the samples were dried under vacuum, fractured in liquid nitrogen, and gold coated before observation. Cross-sectional SEM pictures of hollow fiber membranes were also obtained. Atomic force microscopy (AFM) images were taken in tapping mode with an AFM (Pico Scan 2500). Water contact angles were also measured using a contact angle analyzer (DSA 100, Germany).

\section{Results and Discussion}

To monitor the film growth clearly by UV-vis spectroscopy, multilayer films were first assembled on planar quartz slides. Figure 2 shows the UV-vis spectra of $\left(\mathrm{PSS}(\mathrm{Co})_{1 / 2} / \mathrm{P} 4 \mathrm{VP}\right)_{n}$ multilayers on a PEI-modified quartz substrate $(n=1-10)$. The absorption peaks at 226 and $256 \mathrm{~nm}$ are attributed to $\mathrm{PSS}(\mathrm{Co})_{1 / 2}$ and P4 VP, respectively. The linear increase in absorbance at 226 and $256 \mathrm{~nm}$ as a function of the number of bilayer pairs indicates the progressive deposition process of PSS$(\mathrm{Co})_{1 / 2} / \mathrm{P} 4$ VP films. ${ }^{13} \mathrm{Co}^{2+}$-mediated self-assembly results in a red-brown coloration of the film. ${ }^{43} \mathrm{~A}$ control system of PSS/P4 $\mathrm{VP}$ without $\mathrm{Co}^{2+}$ was used for comparison. It showed a plateau in the absorbance after only five bilayers, indicating that $\mathrm{Co}^{2+}$ is essential to the consecutive deposition of PSS and P4 VP layers and the formation of $\mathrm{PSS}(\mathrm{Co})_{1 / 2} / \mathrm{P} 4 \mathrm{VP}$ multilayers. Using a

(43) Kurth, D. G.; Schütte, M.; Wen, J. Colloids Surf., A 2002, 198, 633. profilometer, we calculated film thicknesses of 21 and $35 \mathrm{~nm}$ for the 6- and 10-bilayer systems, respectively. Therefore, the average bilayer thickness was approximately $3.5 \mathrm{~nm}$. These results confirm that the $\operatorname{PSS}(\mathrm{Co})_{1 / 2} / \mathrm{P} 4 \mathrm{VP}$ films can be successfully built up on a planar substrate and the thickness increment can be controlled on the nanometer scale.

To understand further the role of $\mathrm{Co}^{2+}$ and the driving force for building up films, FTIR spectroscopy of PSS(Co $)_{1 / 2}$, P4 VP, and $\left(\mathrm{PSS}(\mathrm{Co})_{1 / 2} / \mathrm{P} 4 \mathrm{VP}\right)_{25}$ films on $\mathrm{CaF}_{2}$ slides was performed. Because the pyridine ring modes are useful in monitoring intermolecular interactions, FTIR studies could provide evidence of complex interactions. The aromatic carbon-nitrogen stretching band of the pyridine ring at $1602 \mathrm{~cm}^{-1}$ was displaced to a higher wavenumber of $1604 \mathrm{~cm}^{-1}$ (Figure S1 in Supporting Information). Furthermore, the $\mathrm{C}-\mathrm{C}$ stretching band of $1415 \mathrm{~cm}^{-1}$ also gradually shifted to a higher wavenumber of $1422 \mathrm{~cm}^{-1}$. These shifts arise from an increase in the rigidity of the pyridine ring due to intermolecular interactions. ${ }^{44}$ More importantly, the appearance of a new stretching band at $1656 \mathrm{~cm}^{-1}$ for the complexes indicated the formation of nitrogen-metal coordination bonds between pyridine and $\mathrm{Co}^{2+}$-exchanged sulfonate. Actually, the PVP-cobalt complex has been described as tetrahedrally coordinated. ${ }^{45,46}$ XPS results (Figure 3 ) also confirmed the presence of $\mathrm{Co}$ and $\mathrm{S}$ at binding energies of 782 and 168 $\mathrm{eV}$ in the multilayer, respectively. On the basis of the above UV-vis, FTIR, and XPS analyses, it could be concluded that the coordination between pyridine and $\mathrm{Co}^{2+}$-exchanged sulfonate is linked to polymers and in turn formed metal-ligand-coordinated multilayers.

The SEM images of metal-ligand-coordinated multilayers clearly showed that the polyelectrolyte cluster was formed on a quartz slide (Figure S2 in Supporting Information) because of the formation of a polyelectrolyte complex. The topography of the coated film was also evaluated by AFM (Figure S3 in Supporting Information). The dependence of the mean roughness (Ra) and the contact angles (CA) of the multilayer as a function of bilayer number is shown in Figure 4. It was noted that the surface roughness increased with the increase in the number of deposition cycles because of the surface enrichment of metal-ligand-coordinated multilayers. The water contact angle also gradually increased as the number of deposition cycles increased.

It is anticipated that polymer-based dense separation membranes will contribute to a number of important environmental and energy technologies including fuel ethanol manufacturing, hydrocarbon separation, and water desalination. It is also recognized that the creation of new membrane materials is required for these applications. LbL self-assembly is a well-established and very versatile method for constructing vertically structured multilayers on different substrates. For the purpose of separation applications, a $\mathrm{PSS}(\mathrm{Co})_{1 / 2} / \mathrm{P} 4$ VP-coordinated multilayer was then constructed onto flat sheet and hollow fiber polymeric porous substrates, respectively. Different from the rigid substrates of quartz slides and silicon wafers, the polymeric UF membrane substrate has a great number of nanoscale pores and therefore good permeability under the pressure-driven process. Furthermore, UF membrane substrates have a relatively wide pore diameter distribution. In view of the above unique features of UF membrane substrates, dynamic pressure-driven $\mathrm{LbL}$ assembly had recently been developed in our laboratory. ${ }^{21,22,24-26}$ This technique provides a particularly effective approach to

(44) Zhou, X.; Goh, S. H.; Lee, S. Y.; Tan, K. L. Appl. Surf. Sci. 1998, 126, 141. (45) Belfiore, L. A.; McCurdie, M. P.; Ueda, E. Macromolecules 1993, 26, 6908. (46) Ramos, J.; Millán, A.; Palacio, F. Polymer 2000, 41, 8461. 


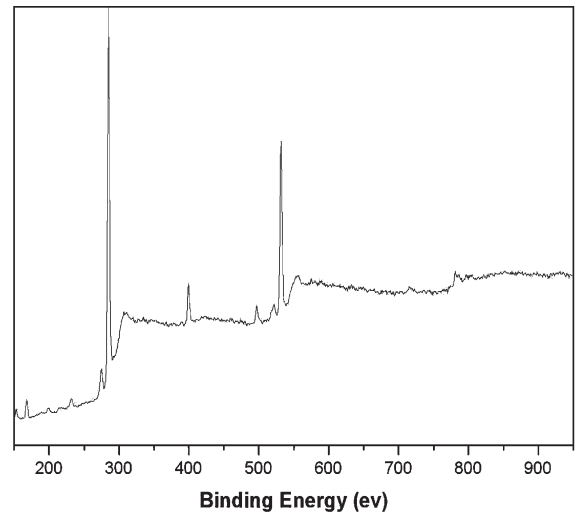

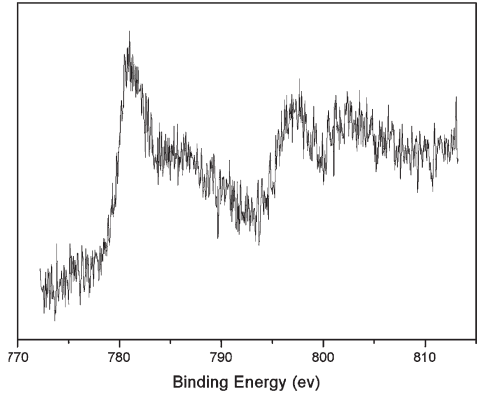

(b) (a)

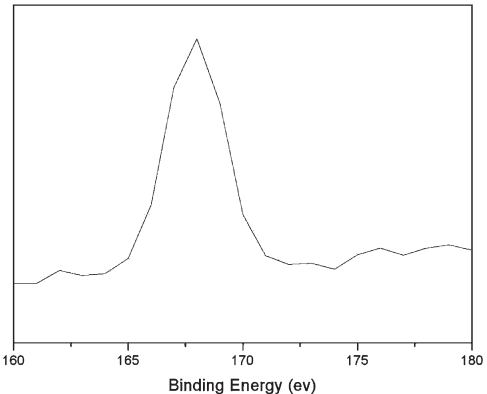

(c)

Figure 3. (a) XPS spectrum of $\left(\mathrm{PSS}(\mathrm{Co})_{1 / 2} / \mathrm{P} 4 \mathrm{VP}\right)_{8}$ multilayer films. (b) Partial enlargement XPS spectrum for Co. (c) Partial enlargement XPS spectrum for $\mathrm{S}$.

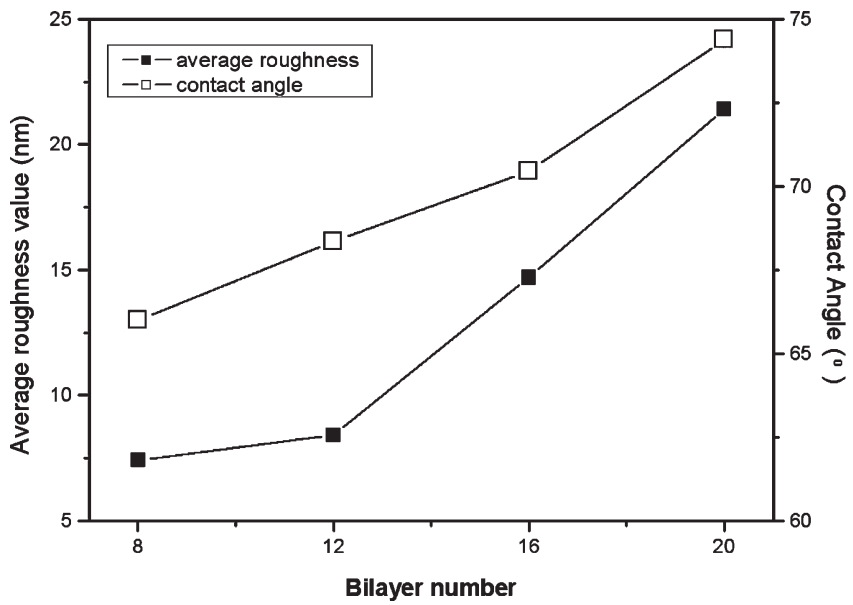

Figure 4. Contact angle and rms roughness of multilayers assembled on planar quartz substrates as a function of bilayer number.

fabricating an ultrathin selective layer by dynamically depositing polymer building blocks onto porous substrates. Each added layer is used to cover the defects in prior layers. In the present work, the PSS $(\mathrm{Co})_{1 / 2}$ and P4 VP solutions were alternatively filtered under a certain transmembrane pressure and consecutively deposited onto a hydrolyzed PAN UF substrate. The membrane surface morphologies before and after dynamic LbL assembly were compared by SEM (Figure 5). For the hydrolyzed PAN UF membrane substrate, a nanoporous surface was clearly observed in Figure 5a. As a comparison, the surface became denser and more uniform after LbL assembly with two bilayers of $\mathrm{PSS}(\mathrm{Co})_{1 / 2} \mathrm{P} 4 \mathrm{VP}$ (Figure $5 \mathrm{~b}$ ). In particular, no appreciable pores could be observed on the top surface, which indicated that a defect-free selective layer was successfully formed. The pervaporation performance of resulting membranes was evaluated using different solvent-water mixtures. The results are shown in Figure 6. It was noted that the membranes showed excellent water-pervaporation performance within only two bilayers, owing to the formation of metal-ligand-coordinated multilayers on the porous substrate. For example, in the case of separating a 95.0 wt $\%$ ethanol-water mixture, the water content could be enriched from $5.0 \mathrm{wt} \%$ (in the feed) to $99.6 \%$ (in the permeate), which meant that the separation factor $(\alpha)$ could reach 4731 whereas the permeate flux was $248 \mathrm{~g} /\left(\mathrm{m}^{2} \mathrm{~h}\right)\left(60^{\circ} \mathrm{C}\right)$, respectively. The selectivity was much higher than those values obtained from electrostatically LbL-assembled polyethyleneimine/poly(acrylic acid) (PEI/PAA) multilayer membranes. ${ }^{21,22}$ Moreover, traditional static $\mathrm{LbL}$ assembly is a time-consuming process that usually requires as many as 50-60 bilayers to obtain defectfree dense membranes. ${ }^{20,23,27}$ In this study, only two $\operatorname{PSS}(\mathrm{Co})_{1 /}$ ${ }_{2} \mathrm{P} 4 \mathrm{VP}$ coordination bilayers that were assembled showed high water-pervaporation selectivity, which showed that dynamic pressure-driven LbL assembly could greatly reduce the number of deposition cycles. Meanwhile, it was suggested that the selectivity increased with the increasing molecular size of alcohols. Similar phenomena have been observed in other types of polyelectrolyte multilayer membranes. ${ }^{22,25}$ It is notable, besides the alcohol-water mixture, that this membrane can be used to dehydrate other solvent-water mixtures. For instance, in the case of the pervaporation of a $95.0 \mathrm{wt} \%$ ethyl acetate-water mixture, the water content in the permeate and permeate flux reached $99.9 \%(\alpha=18981)$ and $1936 \mathrm{~g} /\left(\mathrm{m}^{2} \mathrm{~h}\right)$, respectively. The water-tolerance capacity of the $\left(\mathrm{PSS}(\mathrm{Co})_{1 / 2} \mathrm{P} 4 \mathrm{VP}\right)_{2} / \mathrm{PEI} / \mathrm{PAN}$ membrane was also investigated by changing the water content in the feed solution from 5 to $40 \mathrm{wt} \%$. The results in Figure 7 indicate that the water content in the permeate varied within 


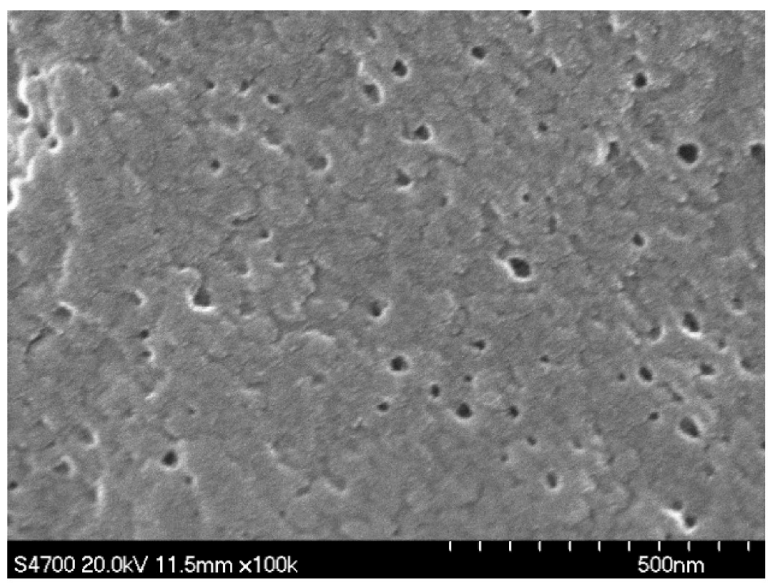

(a)

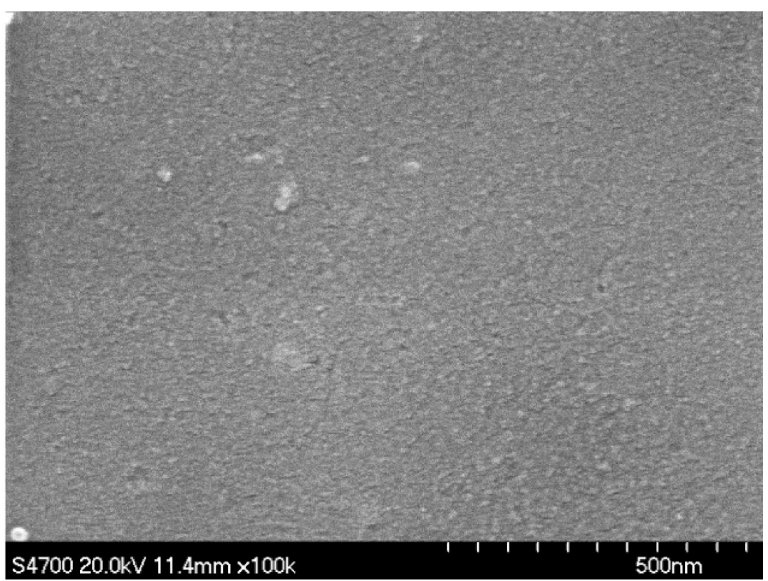

(b)

Figure 5. SEM images of the flat sheet $\left(\mathrm{PSS}(\mathrm{Co})_{1 / 2} / \mathrm{P} 4 \mathrm{VP}\right)_{2} / \mathrm{PEI} / \mathrm{PAN}$ membrane assembled by a dynamic pressure-driven LbL technique: (a) SEM of the top surface before assembly $(\times 100.0 \mathrm{~K})$; (b) SEM of the top surface after assembling two bilayers $(\times 100.0 \mathrm{~K})$.

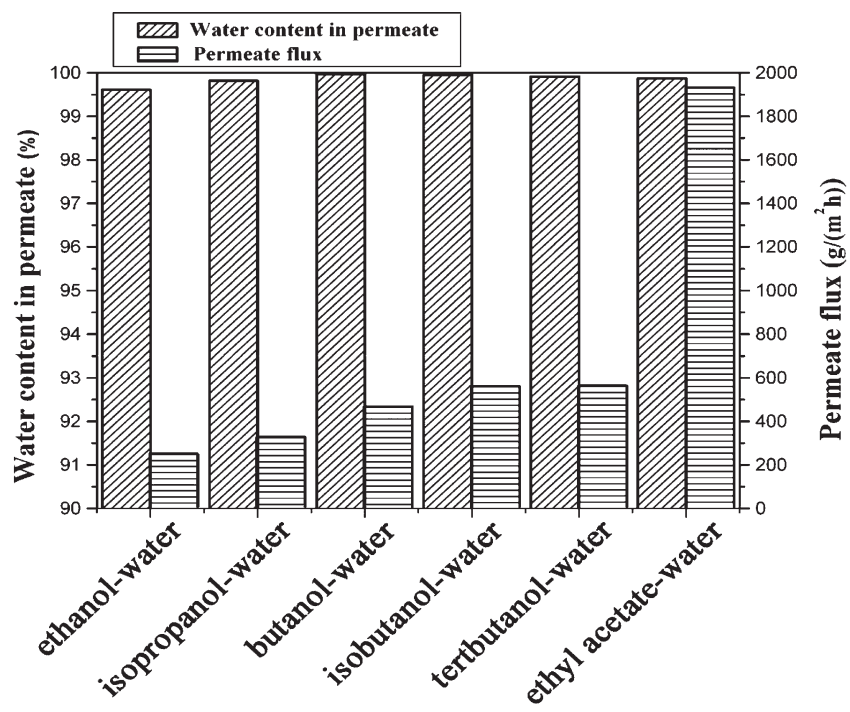

Figure 6. $\left(\mathrm{PSS}(\mathrm{Co})_{1 / 2} / \mathrm{P} 4 \mathrm{VP}\right)_{2} / \mathrm{PEI} / \mathrm{PAN}$ membrane performance for the pervaporation separation of $5 \mathrm{wt} \%$ solvent solutions at $60{ }^{\circ} \mathrm{C}$.

a very narrow band. Even the water content in the feed solution reached $40 \mathrm{wt} \%$, and the corresponding water content in the permeate was maintained at $>99.1 \mathrm{wt} \%$. Compared with our previously reported PEI/PAA multilayer membrane, ${ }^{22}$ the metal-ligand-coordinated multilayer membrane had not only a much higher selectivity but also a much stronger water-tolerance capacity. Furthermore, it was also noted that the permeate flux was up to $>4.6 \mathrm{~kg} /\left(\mathrm{m}^{2} \mathrm{~h}\right)$ in the case of $40 \mathrm{wt} \%$ water content in the feed solution. This high value can be attributed to the water permselectivity of a very thin metal-ligand-coordinated multilayer.

It is well known that the separation of hydrocarbons and their removal from various liquid mixtures are important objectives in the chemical and petrochemical industries. Interestingly, the metal-ligand-coordinated multilayer membrane showed a certain separation capability for aromatic-aliphatic hydrocarbons. In this work, the benzene content could be enriched from 50.0 to 88.0 wt $\%(\alpha=7.3)$ whereas the flux was about $150 \mathrm{~g} /\left(\mathrm{m}^{2} \mathrm{~h}\right)$

(47) Schwarz, H. H.; Malsch, G. J. Membr. Sci. 2005, 247, 143.

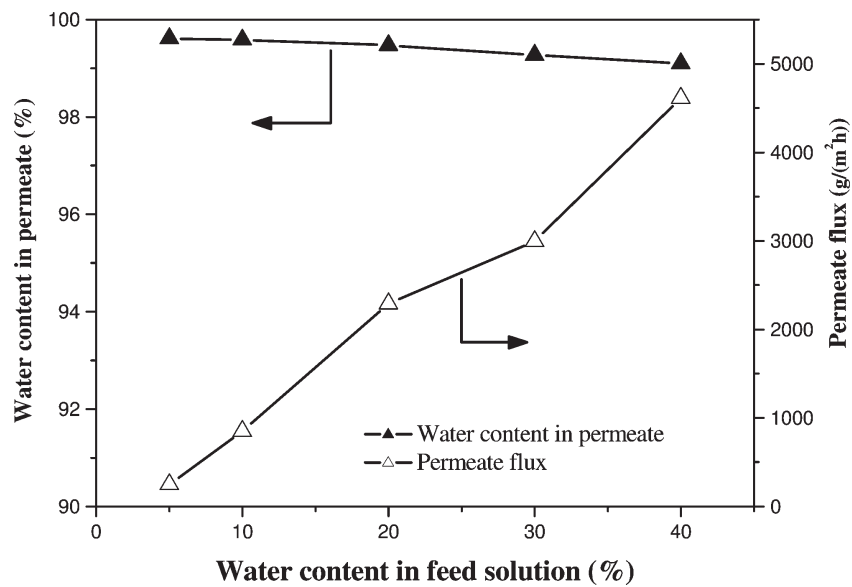

Figure 7. Variation of pervaporation performance with water content in the feed solution at $60{ }^{\circ} \mathrm{C}$.

$\left(70{ }^{\circ} \mathrm{C}\right)$. The selectivity data was comparable to that of the previously reported polyelectrolyte membranes. ${ }^{47}$ Because aromatic molecules have $\pi$-electron clouds, it is commonly recognized that the permselectivity of membranes can be significantly improved with respect to aromatic compounds by introducing special groups with $\pi$-electron acceptors into the polymer. ${ }^{47,48}$ In this case, phenyl and pyridine groups containing multilayers with $\pi$-electron affinity are qualified to form so-called charge-transfer complexes with benzene. Therefore, the PSS $(\mathrm{Co})_{1 / 2} / \mathrm{P} 4 \mathrm{VP}$ multilayer could potentially be selected as a class of new membrane material for the separation of aromatic-aliphatic hydrocarbons. Moreover, the desalination experiments on $\mathrm{MgSO}_{4}$ and $\mathrm{NaCl}$ were performed using the metal-ligand-coordinated multilayer membrane. The permeate flux was determined to be $23.7 \mathrm{~kg} /\left(\mathrm{m}^{2} \mathrm{~h}\right.$ $\mathrm{MPa}$ ). A retention ratio of $95.2 \%$ was achieved for $\mathrm{Mg}^{2+}$ with the $\left(\mathrm{PSS}(\mathrm{Co})_{1 / 2} / \mathrm{P} 4 \mathrm{VP}\right)_{2} / \mathrm{PEI} / \mathrm{PAN}$ membrane. In contrast, the rejection ratio of $\mathrm{Na}^{+}$was only $44.3 \%$. This suggested that the metal-ligand-coordinated multilayer membrane has nanofiltration performance, which is particularly well suited to watersoftening cases.

Although the LbL technique was originally applied for the construction of multilayers of various species on planar

(48) Semenova, S. I. J. Membr. Sci. 2004, 231, 189. 


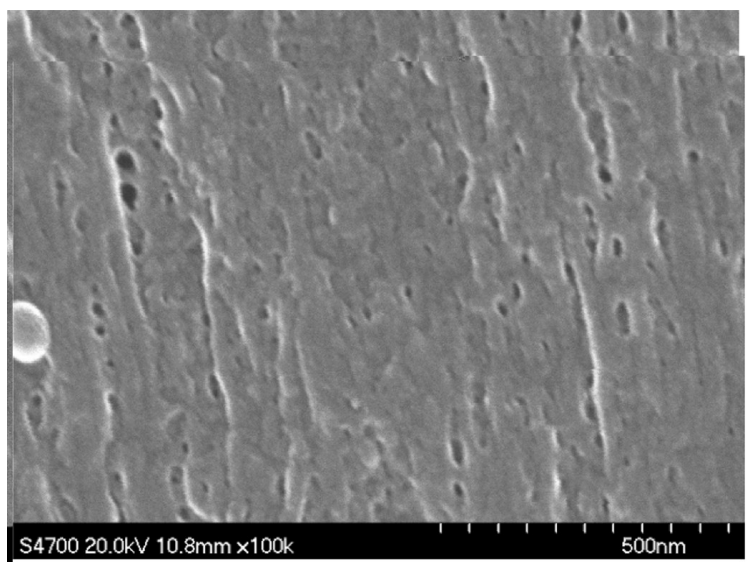

(a)

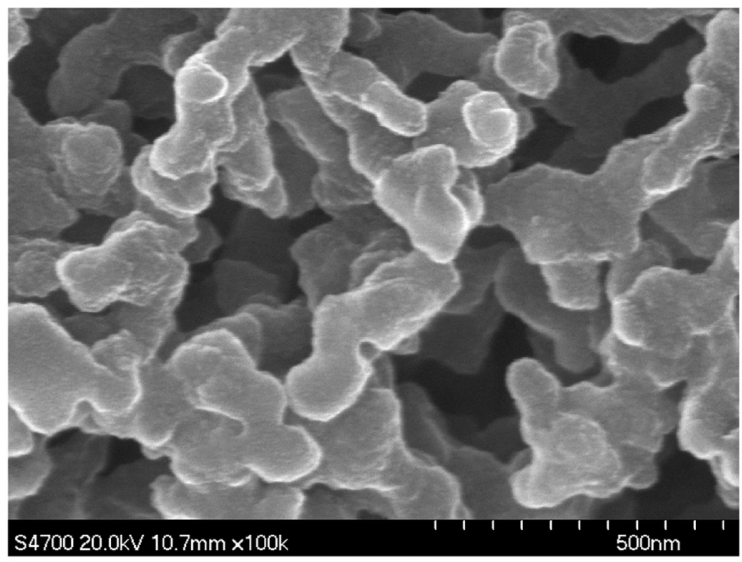

(c)

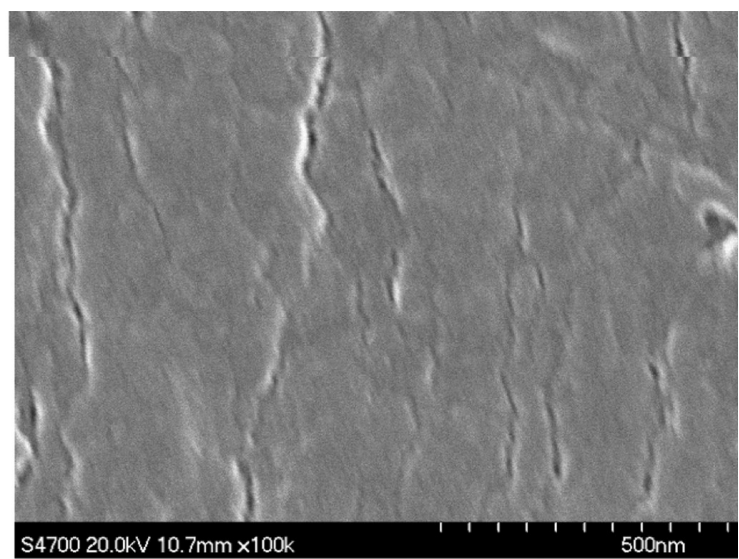

(b)

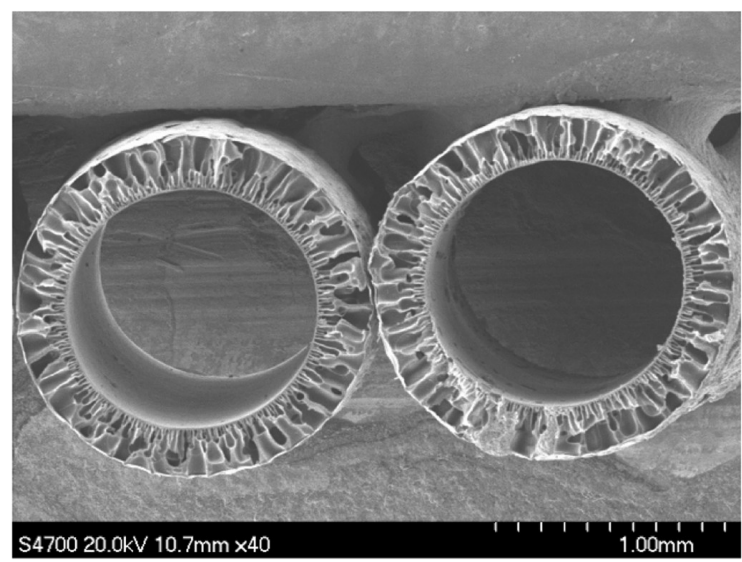

(d)

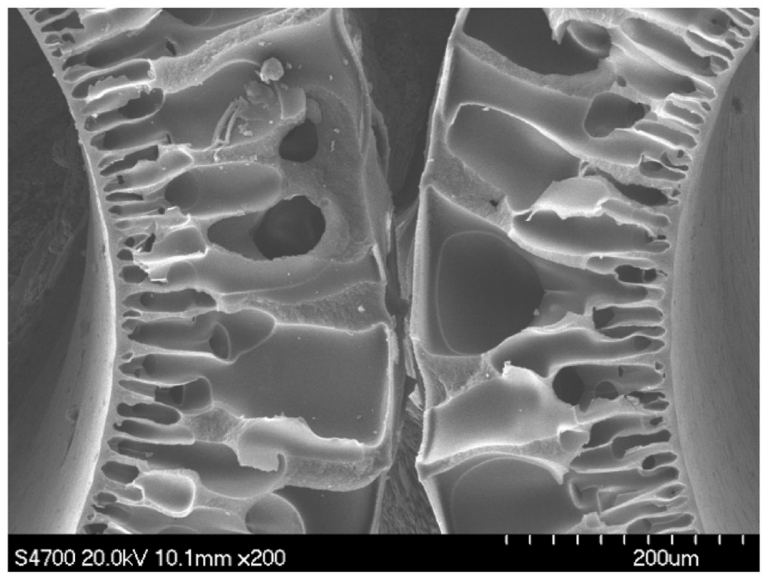

(e)

Figure 8. SEM images of the inner-skin hollow fiber $\left(\mathrm{PSS}(\mathrm{Co})_{1 / 2} / \mathrm{P} 4 \mathrm{VP}\right)_{2} / \mathrm{PEI} / \mathrm{PAN}$ membranes assembled by a dynamic negative-pressure LbL technique: (a) SEM of the inner surface before assembly $(\times 100.0 \mathrm{~K})$; (b) SEM of the inner surface after the assembly of two bilayers $(\times 100.0 \mathrm{~K})$; (c) SEM of the inner surface after the assembly of two bilayers $(\times 100.0 \mathrm{~K})$; (d) overall cross-sectional SEM after the assembly of two bilayers $(\times 40.0 \mathrm{~K})$; and $(\mathrm{e})$ cross-sectional SEM after the assembly of two bilayers $(\times 200.0 \mathrm{~K})$.

substrates, recently scientists have found wide applications in the coating of 3-D substrates. Hollow fiber membranes have some well-known advantages such as high packing density, self-contained mechanical substrates, and hence the consequent economic superiority. In particular, the inner-skin hollow fiber membrane is a unique, promising pervaporation module because it may offer advantages such as easy protection of the defect-free separation layer, good distribution of feed solution, and low transport resistance on the permeate side. ${ }^{24-26}$ However, the construction of a defect-free selective layer on the inner surfaces of hollow fiber substrates still remains an issue. Herein, we present a simple dynamic negative-pressure LbL process for the fabrication of a 
polyelectrolyte complex coating that requires only two bilayers of $\mathrm{PSS}(\mathrm{Co})_{1 / 2} / \mathrm{P} 4$ VP. Outstanding separation capability could be achieved by constructing a metal-ligand-coordinated multilayer on a hollow fiber porous substrate. In the pervaporation of a 95.0 wt $\%$ ethanol-water mixture, the water content in the permeate could reach $99.5 \%(\alpha=3781)$ whereas the flux was maintained at about $250 \mathrm{~g} /\left(\mathrm{m}^{2} \mathrm{~h}\right)$. Figure 8 shows SEM images of the top surface and overall cross-section morphologies of the LbL-assembled membranes. By comparing parts a and $b$ of Figure 8, it could be observed that the pores were completely covered after depositing two bilayers by dynamic pressure-driven LbL assembly. Moreover, a very asymmetric structure was also observed in the hollow fiber membrane walls. As illustrated in Figure $8 \mathrm{~b}-\mathrm{e}$, the hollow fiber was composed of an inner dense layer and an outer porous layer that exhibits a fingerlike structure. For such an asymmetric membrane, the porous supporting layer generally provides negligible resistance and the pervaporation process is mainly controlled by LbL-assembled metal-ligand-coordinated multilayers. However, it is difficult to distinguish the thin multilayer film on top of the substrate after the deposition of two bilayers of $\operatorname{PSS}(\mathrm{Co})_{1 / 2} / \mathrm{P} 4$ VP from the cross-sectional SEM images in Figure 8 d,e. The reason was that the metal-ligand-coordinated multilayer was controlled over the nanoscale range.

\section{Conclusions}

In summary, a route to a new range of metal-ligand-coordinated multilayers has been explored on various substrates. The growth process and driving mechanism were strongly demonstrated during the LbL assembly of multilayer films on quartz and $\mathrm{CaF}_{2}$ slides. It was noted that the metal-ligand plays a key role in the formation of a coordination multilayer. Subsequently, the metal-ligand-coordinated multilayer was constructed on porous polymeric substrates using a proposed dynamic LbL assembly method. In particular, the LbL assembly was successfully implemented on a 3-D hollow fiber substrate under a pressuredriven process. Preliminary studies show that the coordination multilayer membrane will contribute to various separation issues, such as the dehydration of solvent-water mixtures, pervaporation separation of aromatic-aliphatic hydrocarbons, and water desalination. This, in turn, makes it possible to use the simple dynamic pressure-driven $\mathrm{LbL}$ technique to create a metalligand-coordinated functional multilayer membrane for separation. We are currently investigating a range of $\mathrm{Cu}^{2+}$ - and $\mathrm{Ag}^{+}$coordinated multilayer membranes with unique separation properties.

Acknowledgment. This study was supported by the National Natural Science Foundation of China (20806001 and 20876003), the National Basic Research Program of China (2009CB623404), the Program for Beijing Excellent Talents (2009D005015000013), the Beijing NOVA Programme (2006B13), the Beijing Municipal Science and Technology Planning Project (Z08000603170801), and the Funding Project for Academic Human Resources Development in Institutions of Higher Learning Under the Jurisdiction of Beijing Municipality (PRH200907105 and PHR201008018).

Supporting Information Available: Additional FTIR spectra and SEM and AFM images. This material is available free of charge via the Internet at http://pubs.acs.org. 